\title{
Experimental Investigation of the Influences of Fluid Properties on Heat Transfer for Spray Cooling
}

\author{
Jessica Kansy', Thomas Kalmbach¹, André Loges ${ }^{1}$, Thomas Wetzel², Achim Wiebelt ${ }^{1}$ \\ ${ }^{1}$ MAHLE International GmbH \\ Stuttgart, Germany \\ Jessica.Kansy@mahle.com; Thomas.Kalmbach@mahle.com; \\ ${ }^{2}$ Institut für thermische Verfahrenstechnik \\ KIT - Karlsruher Institut für Technologie \\ Karlsruhe, Germany
}

\begin{abstract}
With electrification of the power train, spray cooling becomes more and more important for automobile applications and its electronic components. That's why this investigation focuses on heat transfer performance of spray cooling under automobile boundary conditions. These conditions include wall temperatures below boiling temperature of the fluid, small nozzle to target distances, low nozzle pressures and a vertically positioned target. In order to enable direct contact of the fluid with the electronic components, electrically insulating fluids need to be used. The properties of these dielectric fluids vary in a wide range. Hence this investigation puts special emphasis on the influence of fluid properties on spray cooling. As working medium, water and two water-glycerol mixtures at different temperatures were used to imitate viscosities of exemplary dielectric fluids. The investigated Prandtl numbers range between 5 and 328. To measure heat transfer, an experimental setup based on a steady-state measurement principle was used. A Nusselt correlation, depending on Prandtl and Reynolds number is proposed. The measurement results of heat transfer showed an almost linear dependency on mass flow rate, when spray droplets were impinging on the target. For lower Reynolds numbers, where no spray forms, but an impinging jet sustains, lower heat transfer is observed and the linear dependency does not hold. The droplet size seems to have a negligible effect on heat transfer.
\end{abstract}

Keywords: Spray cooling, dielectric fluid, Nusselt correlation, heat transfer.

\section{Introduction}

Electric components have become an important part of automobile applications. With rising performance requirements power losses increase, while more compact design space and higher integration level of components lead to further increased power densities.

To ensure optimal thermal operation conditions efficient cooling methods are required. In this context cooling techniques, where the hot surface is in direct contact with a dielectric cooling medium, come under scrutiny. One of them is spray cooling, which is most preferably applied below boiling temperature for mobile applications.

Important reviews on spray cooling were done by Kim [1] and Kandlikar et al. [2]. Both of them cover a wide spectrum of topics below and above boiling temperature, with significantly fewer investigations available below boiling temperature. Liang and Mudawar [3] summarized heat transfer correlations on spray cooling below boiling temperature till 2017.

These heat transfer correlations show a great dependence on mass flow rate, viscosity and thermal fluid properties as specific heat capacity and thermal conductivity. Some of them also found a dependence on Weber number [4] [5] and the ratio of wall temperature to saturation temperature [4] [5] [6]. Most of the research was done with water [7] [4] [8] or fluor compounds [9]. That is why the Prandtl number was only investigated in a small range. Two paper covering different fluids are Rybicki et al. [10] and Ashwood et al. [9].

Rybicki et al. [10] used water and PF-5052 while spraying upwards and compared their data to research of Mudawar and Valentine [7], who sprayed downwards. They accomplished developing a Nusselt correlation, which covered both data and both fluids with an overall mean absolute error of $13.1 \%$. They stated that the nozzle orientation (upwards and downwards), had no significant influence. 
Ashwood and Shedd [9] used five different fluids (three perfluorinated hydrocarbons and two segregated hydrofluorether) and mixtures of them. They developed a correlation to calculate the heat transfer coefficient directly using mass flow rate, specific heat capacity, thermal conductivity, kinematic viscosity and a nozzle specific dimensional parameter.

Together both experimental investigations covered Prandtl numbers between 2 and 58. But dielectric fluids cover a much wider range of fluid properties. Therefore this work presents an investigation on the effect of fluid properties on heat transfer for spray cooling below boiling temperature for Prandtl numbers between 5 and 328.

The experimental set up consists of a horizontally positioned hollow cone nozzle targeting a vertical surface with $10 \mathrm{~mm}$ distance. To vary the fluid parameters two different water-glycerol mixtures and water at different temperature level have been used. The nozzle pressures ranged between 900 mbar and 3000 mbar.

\section{Experimental Setup And Procedure}

The experimental set up displayed in Fig. 1 was designed to measure the heat transfer coefficient of a spray including the impinging surface, the thin film area, the hydraulic jump and a part of the downward running fluid. It is a steady-state method and based on temperature measurement to calculate heat flow and surface temperature.

The main part of the setup consists of a copper measurement section (2), which can be divided in a cuboid and a truncated pyramid. On the cuboid a ceramic heater (3) linked to a power adapter (4) was applied as a heat source. The larger end of the truncated pyramid is the target surface $\left(A_{\text {wall }}=75 \times 75 \mathrm{~mm}^{2}\right)$ on which a horizontally positioned nozzle (1) is spraying on. The spray area is covered (19) to ensure reproducible temperature and humidity conditions. The mounting of the copper block possesses low thermal conductivity (6) and is additionally thermally insulated $(5,7)$ to guarantee adiabatic conditions on the outside surfaces.

The nozzle is supplied by a fluid circuit consisting of a reservoir (8), filter $(9,12)$, pumps (10 optional, 11), a vent (14) and funnel (20) to recirculate the fluid. To measure the operating parameter the circuit has a Coriolis mass flow meter (13), resistance thermometer (17) and pressure (18) sensor near the nozzle measuring nozzle pressure $\Delta \mathrm{p}_{\mathrm{n}}$.

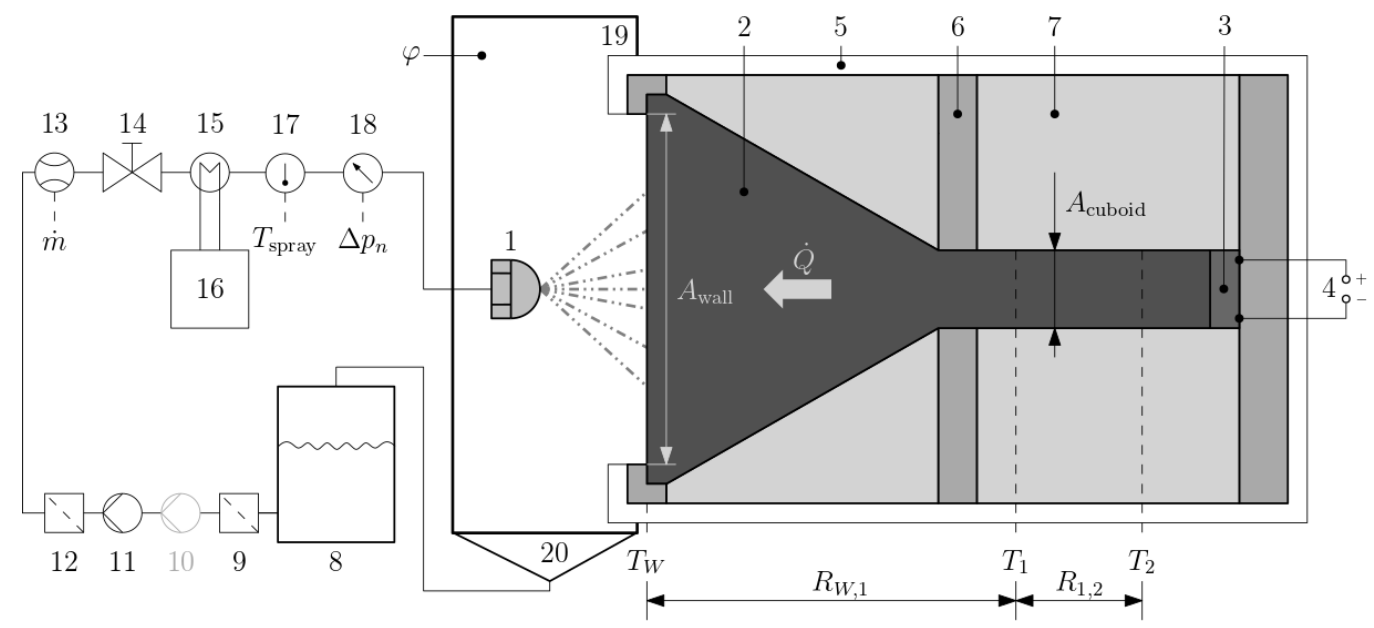

Fig. 1: Experimental set up with spray chamber (1) (19) (20), measurement section (2) - (7), and fluid circuit (8) - (18).

The nozzle type is a hollow cone nozzle with an orifice diameter of $1.4 \mathrm{~mm}$, a spray angle of $120^{\circ}$ and a target distance of $10 \mathrm{~mm}$. Because of the high volume flow the ambient temperature corresponds to the fluid temperature and therefore the spray temperature $\left(\mathrm{T}_{\text {spray }}\right)$. To control this temperature a cooling circuit (16) with heat exchanger (15) has been applied. The small droplet size of the spray ensures a relative humidity $\varphi=100 \%$. Spray temperature and mass flow rate (m) are set variable according to the measurement matrix (Fig. 4). 
With this experimental setup it is possible to determine the heat transfer coefficient $\alpha$ in relation to the target surface $A_{\text {wall }}$ in accordance to the following equation:

$$
\alpha=\frac{\dot{Q}}{A_{\text {wall }}\left(T_{W}-T_{\text {spray }}\right)}
$$

$\dot{Q}$ is the heat flow generated by the ceramic heater and dissipated by the spray. $T_{W}$ is the average wall temperature. Both values have to be calculated by the equations presented in the following:

$$
\begin{gathered}
\dot{Q}=\frac{T_{2}-T_{1}}{R_{1,2}} \\
R_{1,2}=\frac{L}{\lambda_{c u} A_{c u b o i d}} \\
T_{w}=T_{1}-\dot{Q} R_{W, 1}\left(T_{1}, \alpha\right)
\end{gathered}
$$

$T_{1}$ and $T_{2}$ are the temperatures measured in the cuboid by two thermocouples each and $R_{1,2}$ is the corresponding thermal resistance based on the thermal conductivity of copper $\lambda_{\text {cu. }}$. The area of the cross section $A_{\text {cuboid }}$ was chosen to be small compared to the target surface in order to achieve higher temperature differences and reduced measurement uncertainties. The wall temperature $\mathrm{T}_{\mathrm{W}}$ can be determined by $\mathrm{R}_{\mathrm{W}, 1}$ via Fourier's law to heat conduction.

As working medium water-glycerol mixtures were used because their viscosity can be adjusted by temperature and mixing ratio and therefore cover the viscosities of dielectric fluids such as transformer oils and hydrofluorether. Their surface tension is similar to water and they are nontoxic. The fluid properties used in this investigation for these mixtures are based on data of the Glycerine Producers' Association [11].

The viscosities of exemplary dielectric fluids, water and two water-glycerol mixtures between $0{ }^{\circ} \mathrm{C}$ and $40{ }^{\circ} \mathrm{C}$ are shown in Fig. 2. This figure also shows that it is possible to imitate the viscosity of these dielectric fluids by using water and waterglycerol mixtures at different temperatures. The measurement points were chosen based on the following criteria:

- Dynamic viscosity equal to and below $36 \mathrm{mPas}$ : This upper limit results from the spray regime of the used hollow cone nozzle. Above $36 \mathrm{mPas}$ it is not possible to develop a stable hollow cone for nozzle pressures equal and below 3000 mbar nozzle pressure.

- At least three measurement points that represent the viscosity of one dielectric fluid each.

- Temperature variation of water and water-glycerol mixtures: Temperature variation changes the viscosity in a wide range while keeping specific heat capacity and thermal conductivity nearly constant.

The measurement points are marked with a black X. These measurement points cover a viscosity range from $0.7 \mathrm{mPas}$ to $36 \mathrm{mPas}$.

The spray regimes based on Lefebvre [12] of the used hollow cone were analyzed in a preliminary test. The results are shown in Fig. 3. Nozzle pressures between 300 and 3000 mbar and viscosities between 1 and 170 mPas were tested. Above $36 \mathrm{mPas}$ the spray regime changes predominantly from tulip stage (impinging droplets) into onion or jet impingement stage (impinging as connected jet), which was not further considered in this investigation. 


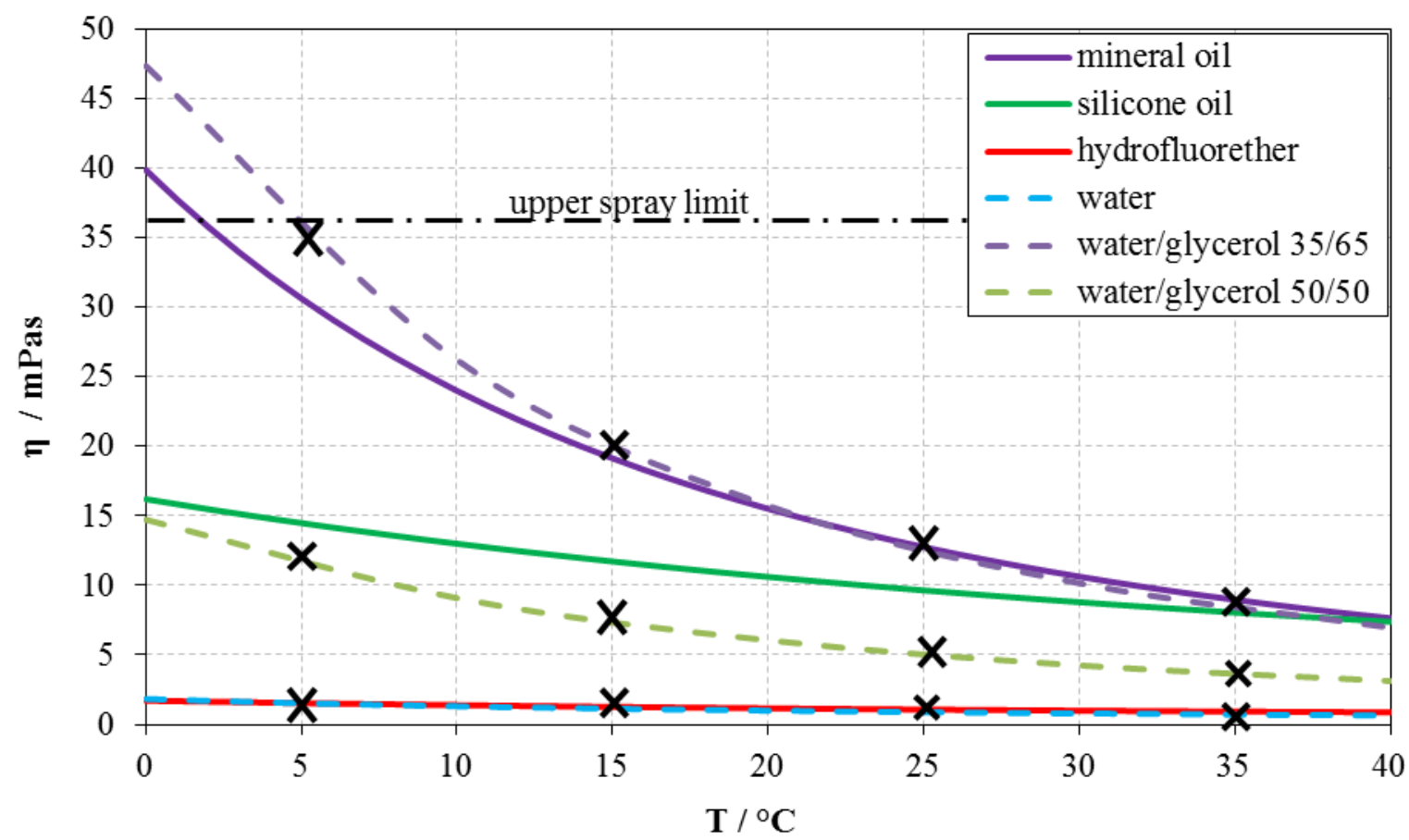

Fig. 2: Dynamic viscosity of three exemplary dielectric fluids, water, two different water-glycerol mixtures and measurement points of the experiments $(\mathrm{X})$.

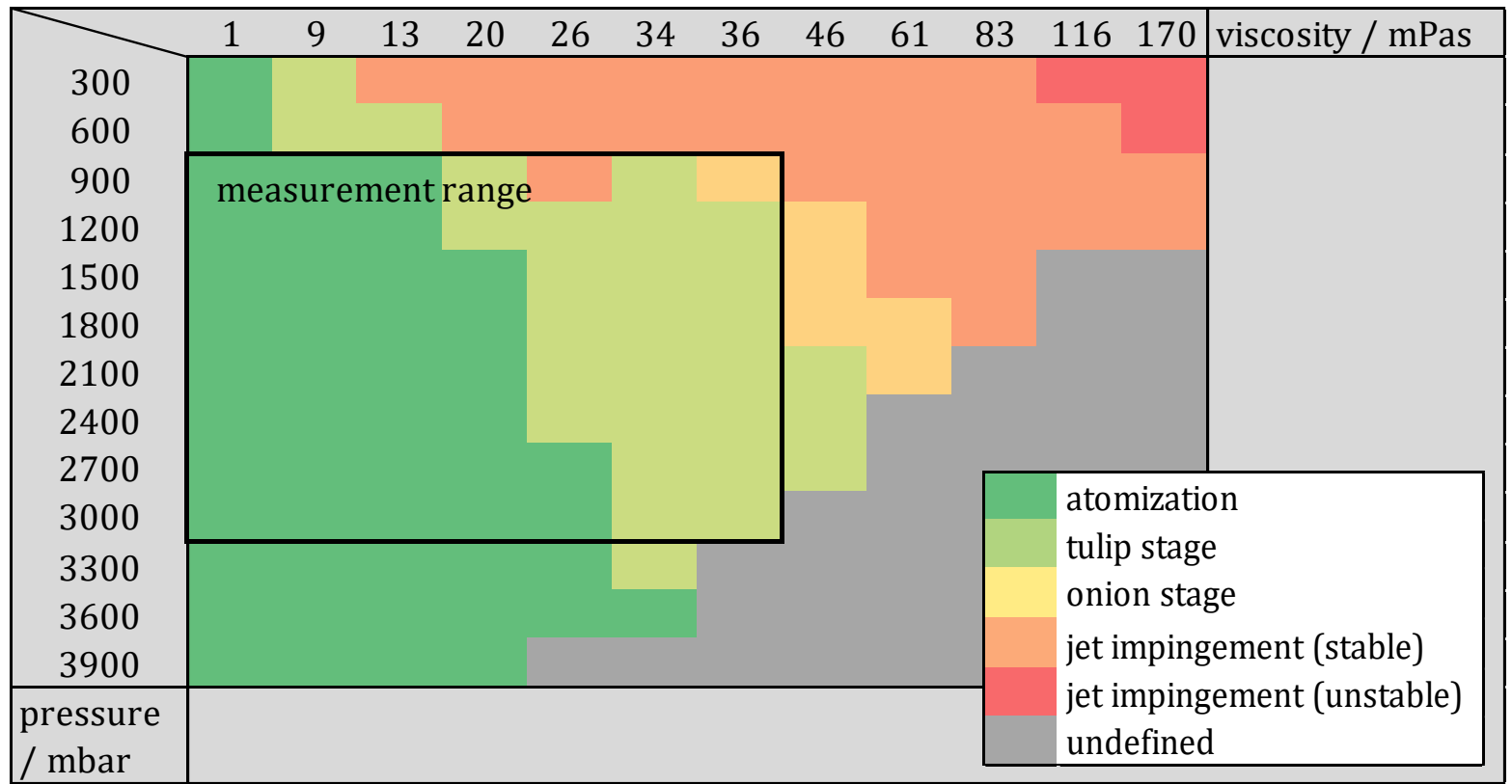

Fig. 3: Spray map of the used hollow cone nozzle using water and water-glycerol mixtures at different viscosities. 


\section{Results and Discussion}

Fig. 4 shows the measured heat transfer coefficients in relation to the heat transfer coefficient measured with water at $35^{\circ} \mathrm{C}$ and a mass flow rate of $25 \mathrm{~kg} / \mathrm{h}$. The measured heat transfer coefficients show a great dependence on mass flow rate and a nearly linear behaviour at low viscosities. Deviations from a linear dependency are especially noticeable at higher viscosities such as water/glycerol-35/65(W/G 35/65) at 35mPas or $20 \mathrm{mPas}$. A comparison of this chart to the spray map in Fig. 3 demonstrates the influence of the different spray regimes on this effect. At high viscosities the fluid impinges as a connected jet cone, whereas at lower viscosities the fluid impinges as droplet cone onto the surface. In the chart the change into an atomized spray results in a higher inclination of the function and therefore in a more efficient heat transfer.

By comparing the heat transfer coefficient of water and water-glycerol mixtures a significant increase becomes obvious. The reasons for this are the more advantageous fluid properties of pure water in comparison to water-glycerol mixtures. They distinguish themselves not just by lower viscosities but also by higher specific heat capacity and thermal conductivity.

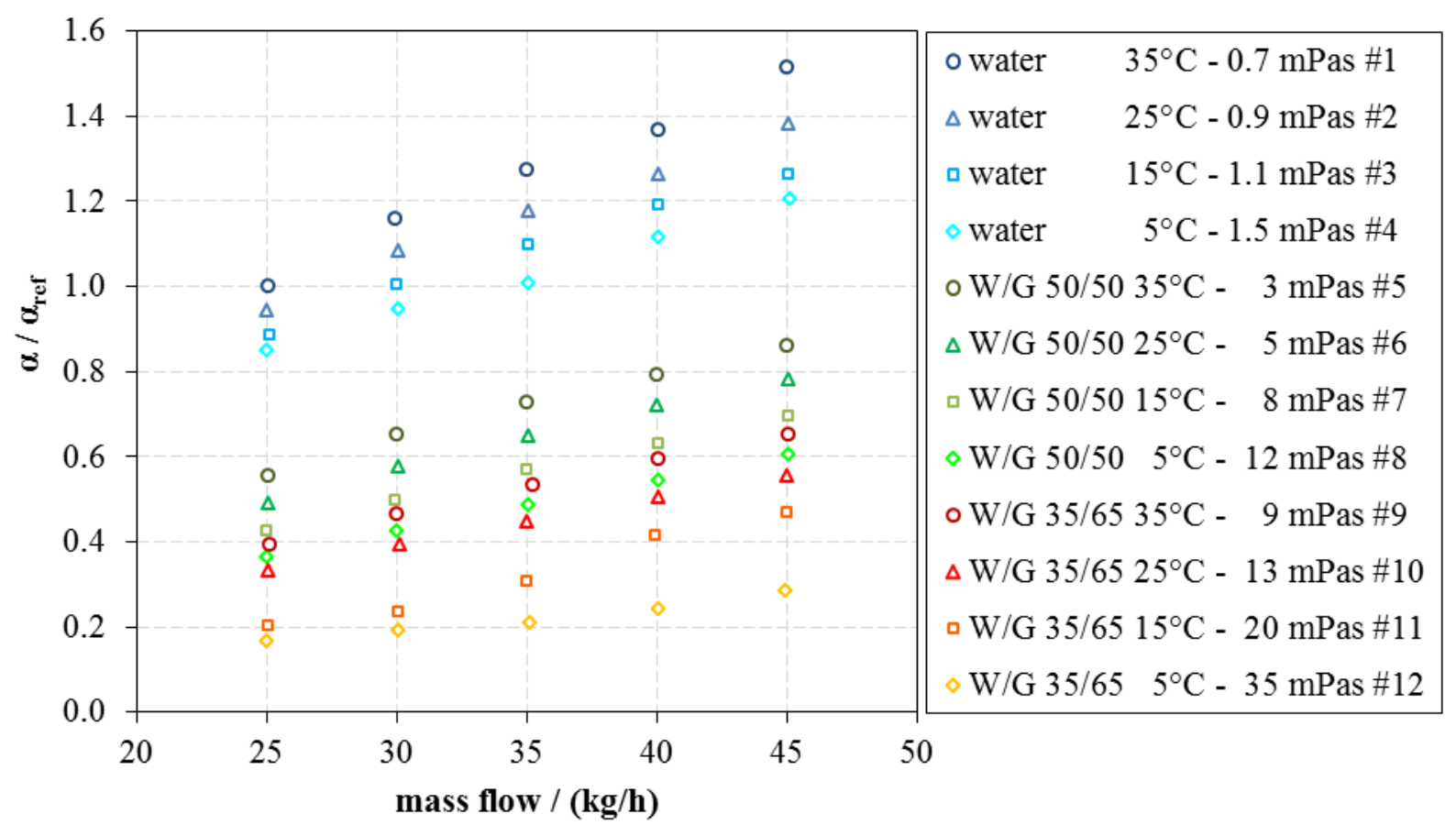

Fig. 4: Measured heat transfer coefficient in relation to reference value (water, $25 \mathrm{~kg} / \mathrm{h}, 35^{\circ} \mathrm{C}, 0.7 \mathrm{mPas}$ ).

To analyse the main influencing factors on heat transfer for spray cooling a dimensional analysis has been made. The main factors can be classified into fluid properties, nozzle type/properties, surface properties, spray properties, environment, geometric parameters and operating parameters. During the experiments some factors have been varied and others have been kept constant. Nozzle, surface, geometrical parameters were kept constant. By changing operating parameters actively spray and environment properties were changed. The fluid properties were varied by using different water-glycerol mixtures.

The dimensional analysis leads to nine independent dimensionless parameters:

$$
\begin{gathered}
f(N u, \operatorname{Pr}, W e, R e, E u, 4 \text { additional geometry related parameters }) \\
\operatorname{Pr}=\frac{\eta c_{p}}{\lambda}, \quad W e=\frac{\rho v^{2} d_{0}}{\sigma}, \quad \operatorname{Re}=\frac{\rho v d_{0}}{\eta}, \quad E u=\frac{\Delta p}{\rho v^{2}}
\end{gathered}
$$


The parameteres are defined by the following variables: dynamic viscosity $\eta$, specific heat capacity $c_{p}$, thermal conductivity $\lambda$, surface tension $\sigma$, density $\rho$, velocity $\mathrm{v}$ of the fluid, nozzle pressure $\Delta \mathrm{p}$ and orifice diameter $\mathrm{d}_{0}$.

The Euler number Eu is mostly nozzle related and varies within a small range. The Weber number We contains the surface tension $\sigma$ of the fluid which barely changed by using different water-glycerol mixtures. Therefore these parameters aren't used for the data point fit for the Nusselt correlation. The parameters in this investigation, which were varied the most, are Prandtl (Pr) and Reynolds (Re) number. Hence these parameters have become the basis of the Nusselt correlation.

The Nusselt correlation is based on the measurement results presented in Fig. 5 and the power-law equation. The exponents and the constant value in front are optimized by the method of least squares.

$$
\begin{gathered}
N u=0.142 \operatorname{Re}^{0.72} \operatorname{Pr}^{0.53} \\
1050<\operatorname{Re}<46500 \\
4.8<\operatorname{Pr}<166
\end{gathered}
$$

In the experiment below $\mathrm{Re}<1050$ fluid impinged as a connected jet cone. The deviation from the presented Nusselt correlation is up to $60 \%$. That is why the lower validity range limit of $\operatorname{Re}$ was set to 1050 . For $\operatorname{Re} \geq 1050$ the fluid impinges as droplets on to the target surface. Within the validity range the maximum deviation of the correlation from the experimental values is $9 \%$ and the mean absolute percentage error is $4 \%$.

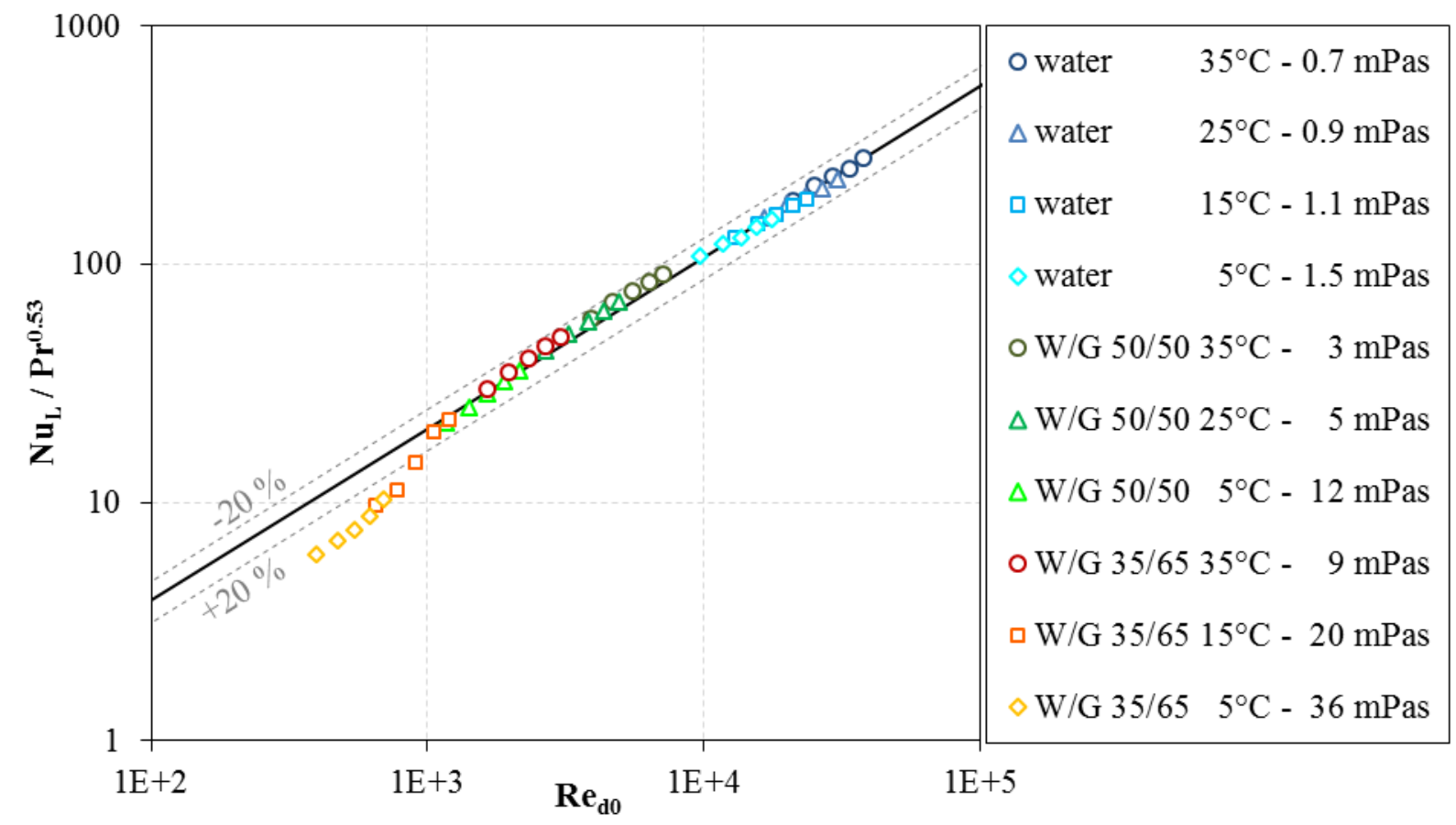

Fig. 5: Nusselt correlation (eq.(7)) and measured Nusselt numbers of the experiment.

Both, the presentations of the experimental results (Fig. 4 and Fig. 5) and the observations regarding the changing spray regime at higher Reynolds numbers confirm a big influence of the way the fluid impinges on the target surface. Droplets impinging on the surface lead to a much higher heat transfer than the impingement of a connected jet.

To evaluate the influence of the different fluid properties on heat transfer, equation (7) has been retransformed into a dimensioned equation (7). 


$$
\alpha=C \rho^{0.36} \eta^{-0.19} c_{p}^{0.53} \lambda^{0.47}
$$

Specific heat capacity and thermal conductivity of the fluid have the highest influence on heat transfer followed by the fluid density. With increasing values of these fluid properties heat transfer increases, whereas an increase of viscosity leads to lower heat transfer. Numerically the fluid viscosity has the lowest influence on heat transfer.

Table 1: Thermophysical fluid properties $\varepsilon_{\mathrm{i}}$ of the water-glycerol mixtures in the experiment of $\mathrm{T}_{\text {spray }}=5^{\circ} \mathrm{C}$ und $\mathrm{T}_{\text {spray }}=35^{\circ} \mathrm{C}$. $\mathrm{The}$ change is calculated by $\varepsilon_{\mathrm{i}}\left(5^{\circ} \mathrm{C}\right) / \varepsilon_{\mathrm{i}}\left(35^{\circ} \mathrm{C}\right)-1$.

\begin{tabular}{|c|c|c|c|c|c|c|c|}
\hline & $\begin{array}{c}\mathbf{T}_{\text {spray }} \\
{\left[{ }^{\circ} \mathrm{C}\right]}\end{array}$ & $\begin{array}{c}\boldsymbol{\eta} \\
{[\mathrm{mPas}]}\end{array}$ & $\begin{array}{c}\boldsymbol{\rho} \\
{[\mathrm{kg} / \mathrm{l}]}\end{array}$ & $\begin{array}{c}\mathbf{c}_{\mathbf{p}} \\
{[\mathrm{kJ} / \mathrm{kg} / \mathrm{K}]}\end{array}$ & $\begin{array}{c}\lambda \\
{[\mathrm{W} / \mathrm{m} / \mathrm{K}]}\end{array}$ & $\begin{array}{c}\boldsymbol{\sigma} \\
{[\mathrm{mN} / \mathrm{m}]}\end{array}$ & $\begin{array}{l}\mathbf{P r} \\
{[-]} \\
\end{array}$ \\
\hline \multirow{3}{*}{$\frac{\grave{ \pm}}{\bar{\pi}}$} & 5 & 1.53 & 1.000 & 4.202 & 0.571 & 74.6 & 11.4 \\
\hline & 35 & 0.72 & 0.944 & 4.182 & 0.615 & 70.2 & 4.95 \\
\hline & Change & $111 \%$ & $0.6 \%$ & $0.5 \%$ & $-7.1 \%$ & $6.2 \%$ & $130 \%$ \\
\hline \multirow{3}{*}{ 突 } & 5 & 111.4 & 1.133 & 3.250 & 0.402 & 70.4 & 995.4 \\
\hline & 35 & 3.61 & 1.118 & 3.310 & 0.421 & 67.0 & 28.8 \\
\hline & Change & $217 \%$ & $1.2 \%$ & $-1.8 \%$ & $-4.5 \%$ & $5.2 \%$ & $231 \%$ \\
\hline \multirow{3}{*}{$\frac{10}{n}$} & 5 & 35.9 & 1.175 & 2.964 & 0.363 & 68.4 & 328 \\
\hline & 35 & 8.34 & 1.159 & 3.049 & 0.371 & 65.7 & 72,3 \\
\hline & Change & $318 \%$ & $1.4 \%$ & $-2.8 \%$ & $-2.0 \%$ & $4.0 \%$ & $354 \%$ \\
\hline
\end{tabular}

Table 1 shows the fluid properties of the different fluid mixtures at $5^{\circ} \mathrm{C}$ and $35^{\circ} \mathrm{C}$. Also the proportional changes of these properties are presented. By comparing these changes it becomes obvious, that the fluid viscosities are changing the most. Despite the numerically low influence of the viscosity it becomes the most important factor.

\section{Conclusion}

The present study investigates the influence of fluid properties on heat transfer for spray cooling below boiling point especially at Prandtl numbers similar to dielectric fluids. With water-glycerol mixtures Prandtl numbers from 5 to 328 were reached. A horizontally positioned hollow cone nozzle with a distance of $10 \mathrm{~mm}$ was targeted on a surface of $75 \times 75 \mathrm{~mm}^{2}$. Besides the investigation on the influence of fluid properties the measured data points have been fitted with a classical power law $\mathrm{Nu}$ approach as a function of Re and Pr.

The measurement results lead to the following statements on the heat transfer for spray cooling:

- At low Reynolds numbers ( $\operatorname{Re}<1050$ for water-glycerol mixtures) the fluid impinges not as droplets, but as connected jet cone.

- Especially for higher Reynolds numbers, when the fluid impinges as droplets, heat transfer is much higher in comparison to the heat transfer of the impinging connected jet cone.

- When fluid impinges as droplets, a linear dependency on mass flow rate is observed

- Changing spray regimes lead to a deviation from the linear dependency.

- Numerically specific heat capacity and thermal conductivity, followed by the fluid density have the highest influence on heat transfer, fluid viscosity the lowest.

- With changing temperature viscosity changes are much higher than changes in specific heat capacity and thermal conductivity. This leads to a much higher influence of fluid viscosity than the rest of the fluid properties. 


\section{References}

[1] J. Kim, "Spray cooling heat transfer: The state of the art," International Journal of Heat and Fluid Flow, vol. 28, pp. 753-767, 2007.

[2] S. G. Kandlikar and A. V. Bapat, "Evaluation of Jet Impingement, Spray and Microchannel Chip Cooling Options for High Heat Flux Removal," Heat Transfer Engineering, vol. 28, pp. 911-923, 2007.

[3] G. Liang and I. Mudawar, "Review of spray cooling - Part 1: Single-phase and nucleate boiling regimes, and critical heat flux," International Journal of Heat and Mass Transfer, vol. 115, pp. 1174-1205, 2017.

[4] W.-L. Cheng, F.-Y. Han, Q.-N. Liu and H.-L. Fan, "Spray characteristics and spray cooling heat transfer in the non-boiling regime," Energy, vol. 36, pp. 3399-3405, 2011.

[5] S.-S. Hsieh and C.-H. Tien, "R-134a spray dynamics and impingement cooling in the non-boiling regime," International Journal of Heat and Mass Transfer, vol. 50, p. 502-512, 2007.

[6] Y. Wang, M. Liu, D. Liu and K. Xu, "Heat Flux Correlation for Spray Cooling in the Nonboiling Regime," Heat Transfer Engineering, vol. 32, pp. 1075-1081, 2011.

[7] I. Mudawar and W. S. Valentine, "Determination of the local quench curve for spray-cooled metallic surfaces," Journal of Heat Treating, vol. 7, pp. 107-121, 1989.

[8] N. Karwa, S. R. Kale and P. M. V. Subbarao, "Experimental study of non-boiling heat transfer from a horizontal surface by water sprays," Experimental Thermal and Fluid Science, vol. 32, pp. 571-579, 2007.

[9] A. C. Ashwood and T. A. Shedd, "Spray Cooling with Mixtures of Dielectric Fluids," in Twenty-Third Annual IEEE Semiconductor Thermal Measurement and Management Symposium, 2007.

[10] J. R. Rybicki and I. Mudawar, "Single-phase and two-phase cooling characteristics of upward-facing and downward-facing sprays," International Journal of Heat and Mass Transfer, vol. 49, pp. 5-16, 2006.

[11] Glycerine Producers' Association, Physical properties of glycerine and its solutions, Gycerine Producers' Association, 1963.

[12] A. Lefebvre, Atomization and Sprays, New York: Hemisphere Publisching, 1989.

[13] Y. Wang, M. Liu, D. Liu, K. Xu and Y. Chen, "Experimental study on the effects of spray inclination on water spray cooling performance in non-boiling regime," Experimental Thermal and Fluid Science, vol. 34, pp. 933$942,2010$. 